\author{
Aneta Tyc
}

\title{
Les conséquences du renversement de la charge de la preuve dans le contexte des arrêts de la Cour de justice de l'Union européenne - Asociaţia Accept (C-81/12) et Galina Meister $(\mathrm{C}-415 / 10)^{1}$
}

\section{Introduction}

Les trois directives sur légalité ${ }^{2}$ ont, en ce qui concerne la charge de la preuve, opté pour un mécanisme qui permet d'alléger, sans la supprimer, ladite charge pour la victime. Le mécanisme mis en place se déroule en deux étapes. En premier lieu, la victime doit alléguer, à suffisance, des faits permettant de présumer l'existence d'une discrimination. Lorsque cette présomption est établie, la charge de la preuve se déplace vers la partie défenderesse. Nous allons principalement nous concentrer sur « les faits qui permettent de présumer l'existence d'une discrimination ».

\section{Arrêt de la Cour dans l'affaire Asociaţia Accept contre Consiliul Naţional pentru Combaterea Discriminării (C-81/12, 25 avril 2013) ${ }^{3}$}

À l'égard de l'exposé des faits, le 3 mars 2010, Accept (organisation non gouvernementale dont l'objet est de promouvoir et de protéger les droits des personnes lesbiennes, gays, bisexuelles et transsexuelles) a déposé plainte (devant la juridiction roumaine) contre

1 Le projet a été financé par des crédits de Centre National pour la Science octroyés sur la base de la décision numéro DEC-2012/05/N/HS5/01589. En 2013, l'auteur a reçu le Prix de la Fondation de l'Université de Lodz.

2 Directive 2000/43/CE du Conseil du 29 juin 2000 relative à la mise en oeuvre du principe de l'égalité de traitement entre les personnes sans distinction de race ou d'origine ethnique, Journal officiel $n^{\circ} \mathrm{L} 180$ du 19/07/2000 p. 0022 '0026; directive 2000/78/CE du Conseil du 27 novembre 2000 portant création d'un cadre général en faveur de l'égalité de traitement en matière d'emploi et de travail, Journal officiel n ${ }^{\circ}$ L 303 du 02/12/2000 p. 0016 '022; directive 2006/54/CE du Parlement européen et du Conseil du 5 juillet 2006 relative à la mise en 'uvre du principe de l'égalité des chances et de l'égalité de traitement entre hommes et femmes en matière d'emploi et de travail (refonte), Journal officiel $n^{\circ}$ L 204 du 26/07/2006 p. 0023 '0036.

$3 \mathrm{http}: / /$ curia.europa.eu/juris/liste.jsf?language=pl\&jur=C,T,F\&num=c-81/12\&td=ALL. 
M. Becali et le SC Fotbal Club Steaua București SA, en faisant valoir que le principe de l'égalité de traitement avait été violé en matière de recrutement. Accept a souligné que, dans le cadre d'une interview concernant l'éventuel transfert d'un footballeur professionnel, X, et l'orientation sexuelle supposée de ce dernier, M. Becali avait tenu, le 13 février 2010, les propos suivants: « Quitte à dissoudre le [FC Steaua], je ne prendrai pas un homosexuel dans l'équipe. Les rumeurs sont des rumeurs, mais écrire ça si ce n'est pas vrai et en plus le mettre en première page... Il (le joueur de football [...] X [...]) n'est peutêtre pas homosexuel. Mais s'il l'est? J'ai dit moi à l'un de mes oncles qui ne croyait ni en Satan ni en Dieu. Je lui ai dit: 'admettons que Dieu n'existe pas. Et s'il existe? Qu'as-tu à perdre à communier? Ça ne serait pas bien d'aller au paradis?' Il m’a donné raison. Un mois avant de mourir, il est allé communier. Que Dieu lui pardonne. Dans ma famille, on ne veut rien avoir à faire avec un gay et le [FC Steaua] est ma famille. Plutôt qu'avoir un gay, mieux vaut que nous jouions avec un joueur de l'équipe junior. Pour moi, ce n'est pas de la discrimination. Personne ne peut m’obliger à travailler avec qui que ce soit. Moi aussi, j'ai le droit de travailler avec qui je veux, tout comme eux ont aussi des droits». Il avait ajouté : «Quitte à dissoudre le Steaua, je ne prendrai pas un homosexuel dans l'équipe! Il n'est peut-être pas homosexuel. Mais s'il l'est? Dans ma famille on ne veut rien avoir à faire avec un homosexuel et le [FC Steaua] est ma famille. Plutôt que d'avoir un homosexuel sur le terrain, mieux vaut que nous prenions un joueur de l'équipe junior. Pour moi ce n'est pas de la discrimination. Personne ne peut mobliger à travailler avec qui que ce soit. Moi aussi j'ai le droit de travailler avec qui je veux, tout comme eux ont aussi des droits. Même si Dieu me garantissait à $100 \%$ pendant la nuit que X n'est pas homosexuel, je ne le prendrais pas! Les journaux ont trop écrit sur le fait qu'il était homosexuel. Même si [le club actuel du joueur X] me le donne gratuitement, je ne le prends pas! Il peut être le plus grand bagarreur, le plus grand buveur... s'il est homosexuel, je ne veux plus entendre parler de lui ».

Accept indiquait que M. Becali avait commis une discrimination directe fondée sur l'orientation sexuelle. À son avis, M. Becali avait violé le principe d'égalité en matière d'embauche et avait porté atteinte à la dignité des personnes homosexuelles. En ce qui concerne la deuxième partie défenderesse devant le CNCD, c'est-à-dire le FC Steaua, Accept a fait valoir que, en dépit du fait que les déclarations de $M$. Becali ont été diffusées dans les médias, à aucun moment ce club de football n'a pris ses distances à l'égard de ces déclarations. Au contraire, le conseil du FC Steaua aurait confirmé que cette politique était menée au niveau du club pour l'engagement des joueurs, au motif que « léquipe est une famille » et que la présence dans celle-ci d'un homosexuel « créerait des tensions dans l'équipe et dans les rangs des spectateurs ». De surcroît, Accept considérait que, au moment où $\mathrm{M}$. Becali avait tenus les propos incriminés, il était encore actionnaire du FC Steaua. 
La Curtea de Apel (Cour d'Appel) Bucureşti avait alors décidé de surseoir à statuer et de poser à la Cour les questions préjudicielles suivantes :

1) Les dispositions de l'article 2, paragraphe 2, sous a), de la [directive 2000/78] sontelles applicables lorsqu'un actionnaire d'un club de football qui se présente lui-même, et est perçu dans les médias comme dans la société, comme étant le principal dirigeant («patron ») dudit club de football fait de telles déclarations dans les médias comme ci-dessus?

2) Dans quelle mesure les déclarations susmentionnées peuvent être qualifiées de « faits qui permettent de présumer l'existence d'une discrimination directe ou indirecte » conformément à l'article 10, paragraphe 1, de la [directive 2000/78], en ce qui concerne la défenderesse [FC Steaua]?

3) Dans quelle mesure est-il ou non question d'une " probatio diabolica " s'il y a retournement de la charge de la preuve, conformément à l'article 10, paragraphe 1 , de la [directive 2000/78], et s'il est demandé à la défenderesse [FC Steaua] d'apporter la preuve du fait qu'il n'y a pas eu violation du principe d'égalité de traitement, en particulier de prouver que « l'orientation sexuelle n'a aucune influence sur l'embauche?».

Selon la Cour de justice, le seul fait que des déclarations, telles que celles en cause au principal, n'émanent pas directement d'une partie défenderesse déterminée (mais provenant de tiers) ne s'oppose pas nécessairement à ce que puisse être établie, au regard de cette partie, l'existence de „faits qui permettent de présumer l'existence d'une discrimination", au sens de l'article 10, paragraphe 1, de ladite directive.

En conséquence, un employeur défendeur ne saurait réfuter l'existence de faits permettant de présumer qu'il mène une politique d'embauche discriminatoire en se limitant à soutenir que les déclarations suggestives de l'existence d'une telle politique d'embauche (en l'occurence homophobe) émanent d'une personne qui, bien quelle affirme et semble jouer un rôle important dans la gestion de cet employeur, n'est pas juridiquement capable de le lier en matière d'embauche.

Dans des circonstances telles que celles du litige au principal, le fait qu'un employeur n'ait pas nettement pris ses distances avec les déclarations en cause constitue un élément dont l'instance saisie peut tenir compte dans le cadre d'une appréciation globale des faits. De surcroît, la Cour a estimé que le fait qu'un club de football professionnel n'ait entamé des négociations en vue du recrutement d'un sportif présenté comme étant homosexuel, n'exclut pas la possibilité que des faits permettant de présumer l'existence d'une discrimination pratiquée par ce club puissent être regardés comme établis.

Selon la jurisprudence de la Cour, la perception du public ou des milieux concernés peuvent constituer des indices pertinents pour l'appréciation globale des déclarations en cause au principal. En ce sens, la Cour a fait référence à l'arrêt dans l'affaire A.G.M.COS.MET Srl contre Suomen valtio, Tarmo Lehtinen ${ }^{4}$. Selon cet aeeêt, sont imputables

4 C-470/03, 17 avril 2007, http://curia.europa.eu/juris/liste.jsf ?language=pl\&jur=C,T,F\&num=c-470/03\&td=ALL. 
à l'État les déclarations d'un fonctionnaire qui, en raison de leur forme et des circonstances, créent chez leurs destinataires l'impression qu'il s'agit de prises de position officielles de l'État, et non pas d'opinions personnelles du fonctionnaire en question. Lélément déterminant pour que les déclarations d'un fonctionnaire soient imputées à l'État réside dans le point de savoir si les destinataires de ces déclarations peuvent raisonnablement supposer, dans un contexte donné, qu'il s'agit de positions que le fonctionnaire prend avec l'autorité de sa fonction. D'après l'avocat général Madame Juliane Kokott, il appartient aux États membres de garantir que leurs fonctionnaires némettent pas, avec l'autorité de leur fonction, de points de vue contredisant la ligne étatique officielle. Si une telle impression venait cependant à naître, elle doit immédiatement être dissipée par un démenti approprié. Dans le cas contraire, le comportement est imputé à l'État, à moins que le fonctionnaire ne soit manifestement incompétent.

En conséquence, comme l'indique la Cour dans l'affaire Asociaţia Accept, il y a lieu de répondre aux première et deuxième questions en considérant que les articles 2 , paragraphe 2, et 10, paragraphe 1, de la directive 2000/78 doivent être interprétés en ce sens que des faits tels que ceux à l'origine du litige au principal sont susceptibles dêtre qualifiés de « faits qui permettent de présumer l'existence d'une discrimination ", en ce qui concerne un club de football professionnel, alors que les déclarations incriminées émanent d'une personne se présentant et étant perçue, dans les médias comme dans la société, comme étant le principal dirigeant de ce club, sans pour autant avoir nécessairement la capacité juridique de le lier ou de le représenter en matière d'embauche. En réalité, et en droit, $M$. Becali aurait vendu les actions qu'il détenait dans le FC Steaua le 8 février 2010 ; cette vente ayant été inscrite au registre du commerce le 23 février suivant, alors que ses propos discriminatoires ont été tenues le 13 février 2010. Cependant, et ce qui est important en droit roumain, la vente d'actions n'est opposable aux tiers qu'à compter de la date à laquelle elle a été rendue publique moyennant sa notification au registre du commerce.

Par sa troisième question, la juridiction de renvoi demande, en substance, si, dans l'hypothèse où des faits tels que ceux à l'origine du litige au principal seraient qualifiés de « faits qui permettent de présumer l'existence d'une discrimination » fondée sur l'orientation sexuelle lors du recrutement des joueurs par un club de football professionnel, la charge de la preuve telle qu'aménagée à l'article 10, paragraphe 1, de la directive 2000/78 ne conduit pas à exiger une preuve impossible à rapporter sans porter atteinte au droit au respect de la vie privée.

La Cour indique que, en ce qui concerne la troisième question, pour renverser la présomption simple dont l'existence peut résulter de l'article 10, paragraphe 1, de la directive 2000/78, il n'est pas nécessaire qu'une partie défenderesse prouve que des personnes d'une orientation sexuelle déterminée ont été recrutées dans le passé, une telle exigence étant effectivement susceptible, dans certaines circonstances, de porter atteinte au droit au respect de la vie privée. 
Dans le cadre de l'appréciation globale qu'il incomberait alors à l'instance nationale saisie d'effectuer, l'apparence de discrimination fondée sur l'orientation sexuelle pourrait être réfutée à partir d'un faisceau d'indices concordants. Ainsi qu'Accept l'a, en substance, fait valoir, parmi de tels indices pourraient notamment figurer une réaction de la partie défenderesse concernée dans le sens d'une prise de distance claire par rapport aux déclarations publiques à l'origine de l'apparence de discrimination ainsi que l'existence de dispositions expresses en matière de politique de recrutement de cette partie aux fins d'assurer le respect du principe de l'égalité de traitement au sens de la directive 2000/78.

Eu égard à ce qui précède, il y a lieu de répondre à la troisième question que l'article 10, paragraphe 1, de la directive 2000/78 doit être interprété en ce sens que, dans l'hypothèse où des faits tels que ceux à l'origine du litige au principal seraient qualifiés de « faits qui permettent de présumer l'existence d'une discrimination » fondée sur l'orientation sexuelle lors du recrutement des joueurs par un club de football professionnel, la charge de la preuve telle qu'aménagée à l'article 10, paragraphe 1, de la directive 2000/78, ne conduit pas à exiger une preuve impossible à rapporter sans porter atteinte au droit au respect de la vie privée.

\section{Arrêt de la Cour dans l'affaire Galina Meister contre Speech Design Carrier Systems GmbH (C-415/10, 19 avril 2012)}

Galina Meister est née en 1961. Elle est d'origine russe. Elle est titulaire d'un diplôme russe d'ingénieur „système” dont l'équivalence avec un diplôme allemand délivré par une Fachhochschule ${ }^{6}$ a été reconnue en Allemagne.

Speech Design a publié une annonce dans la presse afin de recruter un « développeur de logiciels expérimenté $\mathrm{H} / \mathrm{F}$ ». Galina Meister a répondu à cette annonce en se portant candidate le 5 octobre 2006. Par un courrier du 11 octobre suivant, Speech Design a rejeté sa candidature sans la convoquer à un entretien d'embauche. Peu de temps après, une deuxième annonce a été diffusée par Speech Design sur Internet. Le contenu de l'annonce était similaire à celui de la première. Le 19 octobre 2006, Madame Meister s'est portée candidate pour la deuxième fois, mais Speech Design a rejeté sa candidature à nouveau, sans la convoquer à un entretien d'embauche et sans aucunement préciser les motifs de ce rejet.

Aucun des documents détenus par la Cour n'indique que Speech Design a prétendu que le niveau de qualification de Madame Meister ne correspondait pas à celui recherché dans le cadre de cette procédure de recrutement ${ }^{7}$. Estimant qu'elle remplissait les condi-

5 http://curia.europa.eu/juris/liste.jsf ?language=pl\&jur=C,T,F\&num=c-415/10\&td=ALL.

6 Établissement d'enseignement professionnel supérieur.

7 De la même manière, selon M. l'avocat général Paolo Mengozzi, peut être pris en considération le fait que Speech Design ne conteste pas l'adéquation entre le niveau de qualification de Gali- 
tions requises pour occuper le poste en question, Madame Meister a considéré qu'elle a subi un traitement moins favorable qu'une autre personne dans une situation similaire, en raison de son origine ethnique, de son sexe et de son âge. En conséquence, elle a introduit un recours (devant la juridiction allemande) contre la société devant l'Arbeitsgericht, en demandant une indemnisation pour discrimination à l'embauche et qu'elle produise le dossier du candidat recruté ; ce qui lui permettrait de démontrer qu'elle est plus qualifiée que ce dernier.

Madame Meister ayant été débouté de sa demande en première instance, elle a interjeté appel de ce jugement devant le Landesarbeitsgericht, juridiction qui a également rejeté la demande de l'intéressée. Cette dernière a introduit un recours en „Revision” devant le Bundesarbeitsgericht. Cette juridiction s'interroge sur le point de savoir si Madame Meister peut faire valoir un droit à l'information sur le fondement des directives 2000/43, 2000/78 ainsi que 2006/54 et, si tel est le cas, sur les conséquences d'un refus d'information opposé par Speech Design.

Dans cette situation, le Bundesarbeitsgericht a décidé de poser à la Cour les questions préjudicielles suivantes :

«1) L'article 19, paragraphe 1, de la directive 2006/54[...] et l'article 8, paragraphe 1, de la directive 2000/43[...] ainsi que l'article 10, paragraphe 1, de la directive 2000/78[...] doivent-ils être interprétés en ce sens qu'un travailleur alléguant de façon plausible qu'il remplit les conditions d'une annonce de recrutement lancée par un employeur, doit, s'il n'a pas été retenu, pouvoir exiger de cet employeur qu'il lui dise s'il a recruté un autre candidat et, dans l'affirmative, sur la base de quels critères ? En cas de réponse affirmative à la première question :

2) La circonstance que l'employeur ne communique pas les informations demandées constitue-t-elle un fait permettant de présumer l'existence de la discrimination alléguée par le travailleur?"

Conformément à l'arrêt de la Cour, les directives sur l'égalité doivent être interprétées en ce sens qu'ils ne prévoient pas le droit, pour un travailleur alléguant de façon plausible qu'il remplit les conditions énoncées par une annonce de recrutement et dont la candidature n'a pas été retenue, d'accéder à l'information précisant si l'employeur, à l'issue de la procédure de recrutement, a embauché un autre candidat.

Cependant, il ne saurait être exclu qu'un refus de tout accès à l'information de la part d'une partie défenderesse peut constituer l'un des éléments à prendre en compte dans le cadre de l'établissement des faits qui permettent de présumer l'existence d'une

na Meister et celui mentionné dans l'annonce de recrutement ainsi que la double circonstance que, malgré cela, l'employeur ne l'a pas convoquée à un entretien d'embauche et que l'intéressée n'a pas non plus été convoquée dans le cadre de la nouvelle procédure de sélection de candidats pour occuper l'emploi concerné. 
discrimination directe ou indirecte. Il incombe à la juridiction de renvoi, en prenant en considération toutes les circonstances du litige dont elle est saisie, de vérifier si tel est le cas dans l'affaire au principal.

En outre, il faut souligner que, dans l'arrêt Patrick Kelly contre National University of Ireland (University College, Dublin) ${ }^{8}$, la Cour a insisté sur le fait que les instances judiciaires nationales ou les autres instances compétentes doivent prendre en compte les règles de confidentialité découlant des actes du droit de l'Union, tels que la directive 95/46/CE 9 et la directive 2002/58/CE ${ }^{10}$, telle que modifiée par la directive 2009/136/CE du Parlement européen et du Conseil, du 25 novembre 200911. En outre, la protection des données à caractère personnel est également prévue à l'article 8 de la Charte des droits fondamentaux de l'Union européenne.

La Cour dans l'arrêt Kelly a conclu que, si l'article 4, paragraphe 1, de la directive 97/80 ne prévoit pas un droit spécifique en faveur d'une personne qui s'estime lésée par le non-respect à son égard du principe d'égalité de traitement d'accéder à des informations afin qu'elle soit en mesure d'établir des « faits qui permettent de présumer l'existence d'une discrimination directe ou indirecte », conformément à cette disposition, il n'en demeure pas moins qu'il ne saurait être exclu qu'un refus d'information de la part de la partie défenderesse, dans le cadre de l'établissement de tels faits, est susceptible de compromettre la réalisation de l'objectif poursuivi par cette directive et, notamment, de priver ladite disposition de son effet utile ${ }^{12}$.

À la différence de ce qui était le cas dans l'affaire ayant donné lieu à l'arrêt Kelly, l'employeur en cause dans l'affaire au principal semble avoir refusé à Galina Meister tout accès à l'information dont cette dernière sollicite la communication.

8 C-104/10, 21 juillet 2011, http://curia.europa.eu/juris/liste.jsf ?language=pl\&jur=C,T,F\&num=c-104/10\&td=ALL.

9 Directive du Parlement européen et du Conseil, du 24 octobre 1995, relative à la protection des personnes physiques à l'égard du traitement des données à caractère personnel et à la libre circulation de ces données, Journal officiel $n^{\circ}$ L 281 du 23/11/1995 p. 0031 '0050.

10 Directive 2002/58/CE du Parlement européen et du Conseil du 12 juillet 2002 concernant le traitement des données à caractère personnel et la protection de la vie privée dans le secteur des communications électroniques (directive dite de la "vie privée et communications électroniques"), Journal officiel $n^{\circ} \mathrm{L} 201$ du 31/07/2002 p. 0037 '0047.

11 Journal officiel n n L 337 du 18/12/2009 p. 0011 '0036.

12 La directive 97/80/CE (du Conseil du 15 décembre 1997 relative à la charge de la preuve dans les cas de discrimination fondée sur le sexe) a été abrogée et remplacée par la directive 2006/54. Toutefois, eu égard au libellé ainsi qu'à l'économie des articles faisant l'objet du présent renvoi préjudiciel, aucun élément ne permet de penser que, en adoptant les directives 2000/43, 2000/78 et 2006/54, le législateur de l'Union a entendu modifier le régime relatif à la charge de la preuve instauré par l'article 4, paragraphe 1, de la directive 97/80. 


\section{Conclusion}

En résumé, l'arrêt de la Cour dans l'affaire Asociaţia Accept est important en ce qu'il constitue un précédent. Il utilise une approche globale de la question de l'homophobie dans le sport. Il s'agit de lutter contre les discriminations lors du recrutement. Selon la Cour, les déclarations homophobes d'une personne qui est considérée comme étant le dirigeant d'un club de football par le grand public, sans pour autant disposer de la capacité juridique de le lier ou de le représenter en matière d'embauche, peuvent constituer "des faits qui permettent de présumer l'existence d'une discrimination ». Il convient de souligner que les déclarations de M. Becali ont été diffusées dans les médias. À aucun moment, le club de football n'a pris ses distances à l'égard de ces déclarations. En outre, ce club de football n'a engagé aucune négociation en vue du recrutement d'un sportif présenté comme étant homosexuel. Cette circonstance n'exclut pas la possibilité que des faits permettant de présumer l'existence d'une discrimination pratiquée par ce club puissent être regardés comme établis. Cependant, la Cour a interprété largement la législation contre les discriminations. La Cour s'est référée à l'arrêt Feryn ${ }^{13}$ rendu en 2008. Selon la Cour, l'arrêt Feryn ne laisse pas entendre que, pour que l'existence de « faits qui permettent de présumer l'existence d'une discrimination » soit établie conformément à l'article 10, paragraphe 1, de la directive 2000/78, l'auteur des déclarations portant sur la politique d'embauche d'une entité déterminée doit nécessairement avoir la capacité juridique de définir directement cette politique ou encore de le lier ou de représenter cette entité en matière d'embauche.

Dans le secont arrêt (Galina Meister) la Cour a soulevé les problèmes suivants : les directives sur l'égalité ne prévoient pas le droit, pour un travailleur alléguant de façon plausible qu'il remplit les conditions énoncées dans une annonce de recrutement et dont la candidature n'a pas été retenue, d'accéder à l'information précisant si l'employeur, à l'issue de la procédure de recrutement, a embauché un autre candidat. Cependant, il ne saurait être exclu qu'un refus de tout accès à l'information de la part d'une partie défenderesse peut constituer l'un des éléments à prendre en compte dans le cadre de l'établissement « des faits qui permettent de présumer l'existence d'une discrimination directe ou indirecte ».

$13 \mathrm{http}: / /$ curia.europa.eu/juris/liste.jsf ?language=pl\&jur=C,T,F\&num=c-54/07\&td=ALL. 


\section{SUMMARY}

The principle of the reversal of the burden of proof in anti-discrimination cases in the context of the Court of Justice of the European Union C-81/12 and C-415/10

The author of the article refers to two judgements of the Court of Justice of the European Union, which relate to the principle of the reversal of the burden of proof in anti-discrimination cases. She focuses on the facts, from which it may be presumed that there has been discrimination. It cannot be ruled out that a defendant's refusal to grant any access to information may be one of the factors to take into account in the context of establishing facts from which it may be presumed that there has been direct or indirect discrimination (C-415/10). Public statements ruling out the recruitment of a footballer presented as being homosexual are considered to be "the facts from which it may be presumed that there has been discrimination, even though they come from a person presenting himself and being perceived in the media and among the general public as playing a leading role in that club without, however, necessarily having legal capacity to bind it or to represent it in recruitment matters (C-81/12).

KeYwords: Court of Justice of the European Union, judgement C-415/10, judgement C-81/12 
